\title{
Derivation of an Analytical Model for Evaluating the Performance of a Multi- Queue Nodes Network Router
}

\author{
${ }^{1}$ Hussein Al-Bahadili, ${ }^{1}$ Jafar Ababneh, and ${ }^{2}$ Fadi Thabtah \\ ${ }^{1}$ Computer Information Systems \\ Faculty of Information Technology \& Systems \\ The Arab Academy for Banking \& Financial \\ Sciences \\ P.O. Box 13190, Amman 11942, Jordan \\ ${ }^{2}$ Management Information Systems \\ Faculty of Information Technology \\ Philadelphia University \\ P.O. Box 11301, Amman 11123, Jordan \\ ffayez@philadelphia.edu.jo
}

hbahadili@aabfs.org

jafarababneh@yahoo.com

\begin{abstract}
This paper presents a derivation of an analytical model to evaluate the performance of a multi-queue nodes network router, which is referred to as the mQN model. In this model, expressions were derived to calculate two performance metrics, namely, the queue node and system utilization factors. In order to demonstrate the flexibility and effectiveness of the $m Q N$ model in analyzing the performance of a multi-queue nodes network router, two scenarios were performed. These scenarios investigated the variation of queue node and system utilization factors against queue node dropping probability for various numbers of queue nodes, and packets arrival routing probabilities. The performed scenarios demonstrated the flexibility and effectiveness of the $m Q N$ model to evaluate the performance of a multi-queue nodes router prior to the design and implementation stage as compared to experimental tests and computer simulations.
\end{abstract}

KEYWORDS: Congested networks; network routers; active queue management; multi-queue system; analytical modeling; utilization factor.

\section{INTRODUCTION}

Due to the enormous development and wide spread of computer networks and the Internet, congestion becomes a critical issue, which needs to be efficiently solved to achieve optimum network performance $[1,2,3,4]$. Congestion usually occurs at the network router buffer when the number of incoming packets exceeds the available network resources, such as buffer space and processing speed of the network router. Multi-queue systems are widely used as a practical solution to the network congestion problem. Therefore, it is very important to have efficient, reliable, and accurate models to analyze the performance of the network and consequently to contrivance optimum designs for the congestion avoidance queuing system at the network router $[5,6,7,8]$.

Practical experiments and simulations are the most widely-used methodologies for analyzing the performance of multi-queue systems during congestion [2, 8]. However, several efforts have also been directed towards mathematical modeling of multi-queue systems in congested networks. This is because mathematical models are cheaper and easier to use than experimental or simulation applications, and also they can improve understanding of the real problem to set-up appropriate and flexible solutions that suit the network and design requirements $[8,9]$.

Analytical models were developed in $[1,5,6]$ to evaluate the performance of a discrete-time activequeue management (AQM) based queuing systems of one and two-queue nodes; where analytical expressions were derived to calculate a number of performance metrics, such as: average queue length, throughput, average queuing delay, and packet-loss rate. Recently, we have carried-out a research project to extend the model to evaluate the performance of a multi-queue nodes router. 
The research contributed to develop two analytical models and introduced two more performance metrics; these are [10]:

(1) The macroscopic analytical model, which calculates queue nodes and system performance independent of the AQM algorithm, and it is referred to as mQN model. In the $\mathrm{mQN}$ model, expressions were derived to calculate two performance metrics, these are: queue node and system utilization factors, $U_{j}$ and $U_{s}$.

(2) The microscopic analytical model, which calculates the queue nodes performance based on the discrete-time dynamic random early detection (DRED) algorithm. Therefore, it is referred to as DTD-mQN model. In this model, expressions were derived to calculate the four performance metrics mentioned above.

The derived mathematical models can be used to improve understanding of the real problem of a multi-queue nodes router to set-up appropriate and flexible parameters that suit the network and design requirements, and meet all sources (users, services, and applications) needs.

In this paper, we only present the description and derivation of the first model, namely the mQN model. In order to demonstrate the flexibility and effectiveness of the derived expressions, they were used to investigate the effect of the number of queue nodes $(m)$ within the router, and packets arrival routing probability $\left(p_{0 j}\right)$, on $U_{j}$ and $U_{s}$ for various values of dropping probabilities $p_{d}(j)$, where $j=1,2,3, \ldots, m$.

This section provides an introduction to the general domain of this paper. The rest of this paper is organized as follows: Section 2 presents a literature review on the most recent and related researches that concern with developing analytical models to evaluate the performance of queuing systems. Section 3 describes the $m \mathrm{QN}$ model and the derivation of expressions for calculating the performance metrics $U_{j}$ and $U_{s}$. In Section 4, the derived expressions are used to estimate the variation of $U_{j}$ and $U_{s}$ against $p_{d}(J)$ for various values of $m$ and $p_{j 0}$. Finally, in Section 5 , based on the obtained results conclusions are drawn and suggestions and recommendations for future work are pointed-out.

\section{Literature Review}

This section presents a literature review on the most recent and related researches that were conducted to develop analytical performance evaluation models for the existing queuing systems and algorithms. At this stage, it is very important to realize that there are only limited numbers of references precisely concern with developing such analytical models, as most researchers use computer simulations or experimental tests in evaluating their systems or algorithms.

$\mathrm{H}$. Abdel-Jaber et. al [5] derived an analytical model to evaluate the performance of a discrete-time DRED-based queuing system, which consists of one and two-queue nodes. They derived analytical expressions to calculate four performance metrics, these are: average queue length, throughput, average queuing delay, and packet-loss rate.

L. Hoflack et. al [11] derived approximate expressions to calculate the tail probabilities of the buffer occupancy (number of packets stored in the buffer) in a discrete-time queuing model with infinite storage capacity and one single output line. This model applied to study the traffic of a file server, where one file download by a user corresponds to one session. The steady-state probability generating functions of the number of active sessions, buffer occupancy and packet delay were derived. The derived expressions can be used to study the influence of the different system parameters.

F. Baccelli and D. McDonal [12] derived a closed form formula for the stationary throughput obtained by an HTTP flow, which consists of a succession of idle and download periods. The file downloads are subject to a fixed packet-loss probability. For this stochastic model, they also derived closed form expressions for the mean time to transfer a file and for the distribution of the throughput. Several laws of file sizes and idle times were considered including heavy tailed distributions. They briefly discussed how the formulas can be applied to predict bandwidth sharing among competing HTTP flows.

L. Guan et. al [1] presented a discrete-time queuing model for the performance evaluation of congestion control based on Random Early Detection (RED) using queue thresholds. The 
analytical model for a discrete-time finite queue incorporates two thresholds $H_{1}$ and $H_{2}\left(H_{2}>H_{1}\right)$. Before the number of packets in the system reaches the first threshold $H_{1}$, the queue operates normally with arrival rate $\alpha_{1}$. When the number of packets in the system is between $H_{1}$ and $H_{2}$, packets are dropped probabilistically with the drop probability $\left(p_{d}\right)$ increasing linearly with system contents. Beyond $H_{2}, p_{d}$ then remains constant until the number of packets reaches the system capacity. The system can be potentially used as an analytical model for congestion control based on RED.

I. Awan et. al [13] presented a framework for the performance analysis of queuing networks with blocking under AQM scheme. The analysis was based on a queue-by-queue decomposition technique. The use of queue thresholds is a well-known technique generalized exponential distribution which can capture the bursty property of network traffic. The analytical solution was obtained using the maximum entropy principle. The forms of the state and blocking probabilities were analytically established at equilibrium via appropriate mean value constraints. The initial numerical results demonstrated the credibility of the proposed analytical solution.

L. Wang et. al [14] developed an analytical performance model for a finite capacity queuing system with AQM mechanisms based on the instantaneous number of packets in the queue. They considered two different traffic classes modeled by Poisson arrival processes. Expressions for the aggregated and marginal performance measures including the mean queuing length, response time, system throughput, probability of packet-losses and mean waiting time were derived. The validity of the analytical results was demonstrated by comparing the analytical results and those obtained from simulation experiments.

\section{The DTD-mQN Analytical Model}

Queuing systems with more queue nodes are required to meet the evolving network needs. Therefore, it is important to have efficient tools to evaluate the performance of such systems and help system designers to select appropriate design parameters $[5,6,15]$. It is clear that availability of such tools play a crucial role in designing and building cost-effective queuing systems.

This paper is concerned with the development of such tool, in particular, development of an analytical model that can be used to evaluate the performance of a multi-queue nodes queuing system. Thus, it is referred to as $m Q N$ analytical model. Before we proceed with the derivation of the $m Q N$ model, we believe it is important to describe the architecture of the queuing system and how it works, and also to define the system variables. But first, let us start with the definition of the system variables. System variables are classified into design and network variables as follows:

(1) Design-driven variables, which are the variables derived from the technological design of the system, such as: number of queue nodes, buffer size (maximum queue size), and various interaction probabilities.

(2) Network-driven variables, which are the variables derived from the network environment, such as packets arrival rate.

\subsection{The Architecture of the Multi-Queue Nodes System}

The architecture of a multi-queue nodes system consisting of $m$ queue nodes ( $j=1$ to $m$ ) is shown in Figure 1. The architectural model shows that the system consists of main arrival-point, where all packets are arrived there, and then distributed according to certain probabilities $\left(p_{0 j}\right)$ to the different arrival-points of each queue node within the system. Each queue node has one arrival-point and one departure-point. The packets leaving a queue node are either depart the system, feedback to the same queue node, or bypass to other queue nodes within the system, each with a certain probability. In addition, each queue node has a certain packet dropping probability $\left(p_{d}(f)\right)$. 


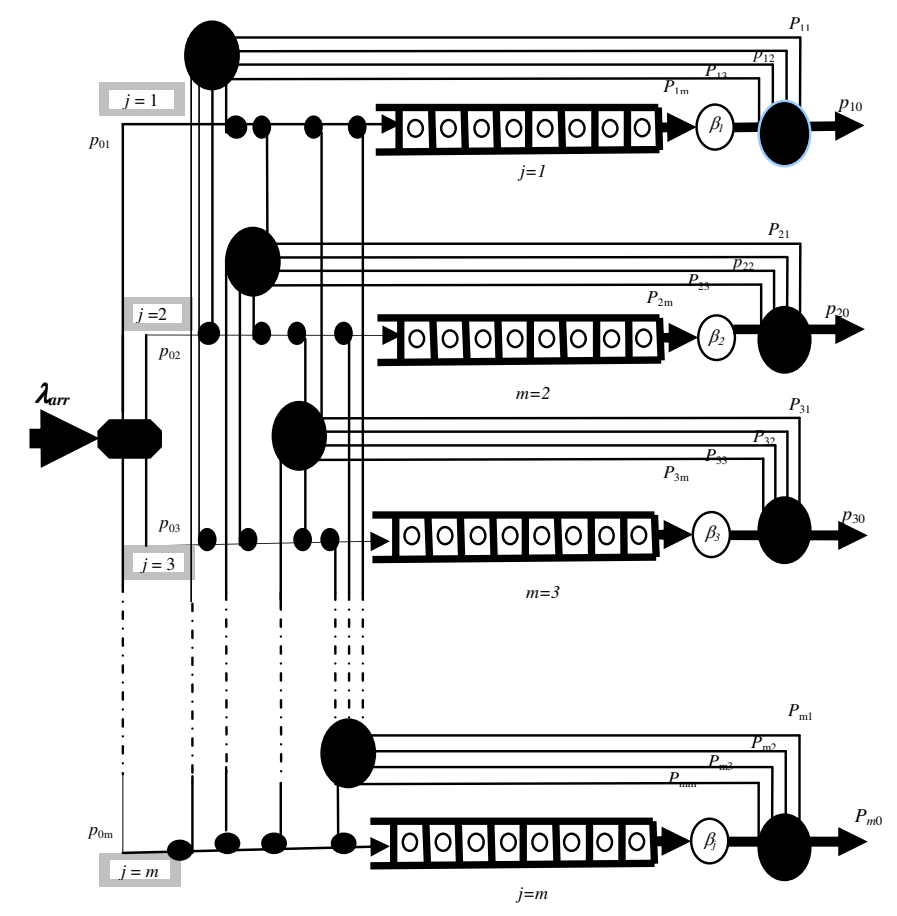

FIGURE 1: The architecture of a multi-queue nodes system.

\subsection{Derivation of the $m Q N$ Analytical Models}

Figure 1 assumes that data packets arrive to the network router at an arrival rate of $\lambda_{\text {arr }}$. Then these data packets are distributed between the queue nodes according to $p_{0 j}$, where $p_{0 j}$ is defined as the probability that a packet arriving at the network router is forwarded to queue node $j$. The actual packet rate reaches the arrival point of queue node $j$ is denoted as $\lambda_{0 j}$ and can be mathematically calculated as:

$$
\lambda_{0 j}=p_{0 j} \lambda_{a r r}
$$

The input to each queue node also includes packets that may be bypassed from other queue nodes in the queuing system, including the queue node itself (i.e., feedback). These packets are bypassed according to pre-adjusted probabilities $\left(p_{i j}\right)$, which can be defined as the probability that a packet is bypassed from queue node $i(i=1$ to $m$ ) to queue node $j(j=1$ to $m$ ).

\section{Calculation of the queue node packets arrival rate $\left(\lambda_{i}\right)$}

It can be seen from Figure 1 that the total packet arrival rate for any queue node $\left(\lambda_{j}\right)$ within the system can be calculated by establishing a packet balance at the arrival-point of queue node. Accordingly, $\lambda_{j}$ can be calculated as:

$$
\lambda_{j}=p_{0 j} \lambda_{a r r}+\sum_{i=1}^{m} p_{i j} B_{i}=\lambda_{0 j}+\sum_{i=1}^{m} p_{i j} B_{i} \quad(j=1 \text { to } m)
$$

Where $p_{0 j}$ is the probability that a packet arriving at the network router arrival-point is bypassed to queue node $j ; p_{i j}$ is the probability that a packet is bypassed from queue node $i$ to $j$ (it is called feedback probability when $i=j$ ), $\lambda_{\text {arr }}$ is the packets arrival rate at the network router (network arrival rate); $\lambda_{j}$ is the total packets arrival rate at queue node $j, B_{j}$ is the queue node departure rate, and $m$ number of queue nodes. The arrival rate of any queue node $j\left(\lambda_{j}\right)$ can also be calculated as: 


$$
\lambda_{j}=B_{j}+\lambda_{d}
$$

Where $\lambda_{d}$ is the dropping rate, which can be calculated as:

$$
\lambda_{d}=p_{d}(J) \cdot \lambda_{j}
$$

Substituting Eqn. (4) into Eqn. (3) yields:

$$
\lambda_{j}=B_{j}+p_{d}(j) \lambda_{j}=\frac{B_{j}}{1-p_{d}(j)}
$$

Substituting Eqn. (5) into Eqn. (2) yields the following general set of linear equations:

$$
\left(p_{j j}-\frac{1}{1-p_{d}(j)}\right) B_{j}+\sum_{\substack{i=1 \\ i \neq j}}^{m} p_{i j} B_{i}=-p_{0 j} \lambda_{a r r} \quad(j=1 \text { to } m)
$$

Eqn. (6) can be simplified to:

$$
\hat{p}_{j j} B_{j}+\sum_{\substack{i=1 \\ i \neq j}}^{m} p_{i j} B_{i}=-p_{0 j} \lambda_{a r r}(j=1 \text { to } m)
$$

Where $\hat{p}_{j j}=p_{j j}-\frac{1}{1-p_{d}(j)}$

Eqn. (7) can be written in a matrix form as:

$$
\left[\begin{array}{cc:ccc}
\hat{p}_{11} & p_{21} & p_{31} & \mathrm{~L} & p_{m 1} \\
p_{12} & \hat{p}_{22} & p_{32} & \mathrm{~L} & p_{m 2} \\
p_{13} & p_{23} & \hat{p}_{33} & \mathrm{~L} & p_{m 3} \\
\mathrm{M} & \mathrm{M} & \mathrm{M} & \mathrm{L} & \mathrm{M} \\
p_{1 m} & p_{2 m} & p_{3 m} & \mathrm{~L} & \hat{p}_{m m}
\end{array}\right]\left[\begin{array}{c}
B_{1} \\
B_{2} \\
B_{3} \\
\mathrm{M} \\
B_{m}
\end{array}\right]=\left[\begin{array}{c}
-p_{01} \lambda_{a r r} \\
-p_{02} \lambda_{a r r} \\
-p_{03} \lambda_{a r r} \\
\mathrm{M} \\
-p_{0 m} \lambda_{a r r}
\end{array}\right]
$$

Eqn. (9) represent a set of $m$ linear equations with $m$ unknowns $\left(B_{j}\right)$ from $j=1$ to $m$. The above set of linear equations can be easily solved using linear solvers, such as: Gaussian elimination [16]. After computing the values of the queue node departure rate $B_{j}$ the values of $\lambda_{j}$ can be computed using Eqn. (5).

Calculation of the queuing system packets departure rate $\left(\lambda_{\text {dep }}\right)$

As we have discussed earlier in this section that packets leaving the queue at any of the queue node within the system are routed in one of the following directions:

(1) Depart from the queuing system with probability $\left(p_{j 0}\right)$.

(2) Feedback to the same queue node with probability $\left(p_{j j}\right)$.

(3) Bypassed to be re-queued at other queue nodes within the system with probability $\left(p_{i j}\right)$.

In all cases $i$ and $j$ varies from 1 to $m$. A packet balance equation can be established at the departure-point of each queue node as follows:

$$
B_{j}=p_{j 0} B_{j}+p_{j j} B_{j}+\sum_{\substack{k=1 \\ k \neq j}}^{m} p_{j k} B_{j}=\sum_{k=0}^{m} p_{j k} B_{j}
$$


Where $B_{j}$ is the departure rate from queue node $j ; p_{j 0}$ is the probability of sending packets outside the queuing system, for example, toward the processing unit; $p_{j j}$ is the probability of bypassing a packet back to be re-queued at the same queue node; $p_{j k}$ is the probability of bypassing packets to other queue nodes within the system. The departure rate of the queue node, i.e., the number of packets departed from each node per unit time, which is referred to as $p_{j 0}$ may be calculated as:

$$
\lambda_{j 0}=p_{j 0} B_{j}
$$

So that the total net departure rate $\left(\lambda_{\text {dep }}\right)$ of the queuing system, i.e., the total number of packets departed from all queue nodes per unit time, is given by:

$$
\lambda_{\text {dep }}=\sum_{j=1}^{m} p_{j 0} B_{j}=\sum_{j=1}^{m} \lambda_{j 0}
$$

\section{Calculation of the queuing system and queue node utilization factors}

Following the derivations of an expression to calculate the queue node and system and arrival and departure rates, it is possible now to calculate the utilization factor for each of them as follows:

(1) Queue node utilization factor $\left(U_{j}\right)$. It is defined as the ratio between the queue node departure rate $\left(B_{j}\right)$ and the queue node arrival rate $\left(\lambda_{j}\right)$, which is mathematically expressed in percentage form as:

$$
U_{j}=\frac{B_{j}}{\lambda_{j}} \times 100
$$

(2) Queuing system utilization factor $\left(U_{s}\right)$. It is defined as the ratio between the queuing system departure rate $\left(\lambda_{\text {dep }}\right)$ and the system arrival rate $\left(\lambda_{\text {arr }}\right)$, which is mathematically expressed in percentage form as:

$$
U_{s}=\frac{\lambda_{\text {dep }}}{\lambda_{\text {arr }}} \times 100
$$

Substituting for $\lambda_{\text {arr }}$ from Eqn. (2) and $\lambda_{\text {dep }}$ from Eqn. (12) into Eqn. (14), $U_{s}$ can also be expressed as:

$$
U_{s}=\sum_{j=1}^{m} p_{o j} p_{j o} \frac{B_{j}}{\lambda_{j}}=\sum_{j=1}^{m} p_{o j} p_{j o} U_{j}
$$

\section{RESULTS AND DISCUSSIONS}

The $m Q N$ analytical model is implemented in a $\mathrm{C}++$ code, and used to evaluate the performance of a multi-queue node system. It can be recognized that there are infinite number of scenarios that can be performed using different combinations of network and design parameters, and for each scenario the performance of the queue node and/or the system can be investigated. Investigating the performance for all possible combinations is impractical and beyond the objectives of this paper. In this paper two scenarios are implemented. These scenarios investigated the variation of $U_{j}$ and $U_{s}$ against $p_{d}(J)$ for various values of $m$ and $p_{0 j}$.

\subsection{Scenario \#1. Investigate the Variation of $U_{j}$ and $U_{s}$ against $p_{d}(J)$ for Various Values of $\boldsymbol{m}$}

In this scenario, the mQN model is used to investigate the variation of $U_{j}$ and $U_{s}$ against $p_{d}(l)$ for various values of $m(m=3,4$, and 5$)$. The values of $p_{d}(j)$ vary between 0 to 0.25 in step of 0.05 and $\lambda_{\text {arr }}$ is unity. The queue nodes are assumed to have equal arrival and departure routing probabilities, and, the feedback routing probability is always twice the bypass routing probabilities. 
Table 1 lists the queue nodes packets arrival, feedback, bypass, and departure routing probabilities.

TABLE 1: Packets routing probabilities for Scenario \#1.

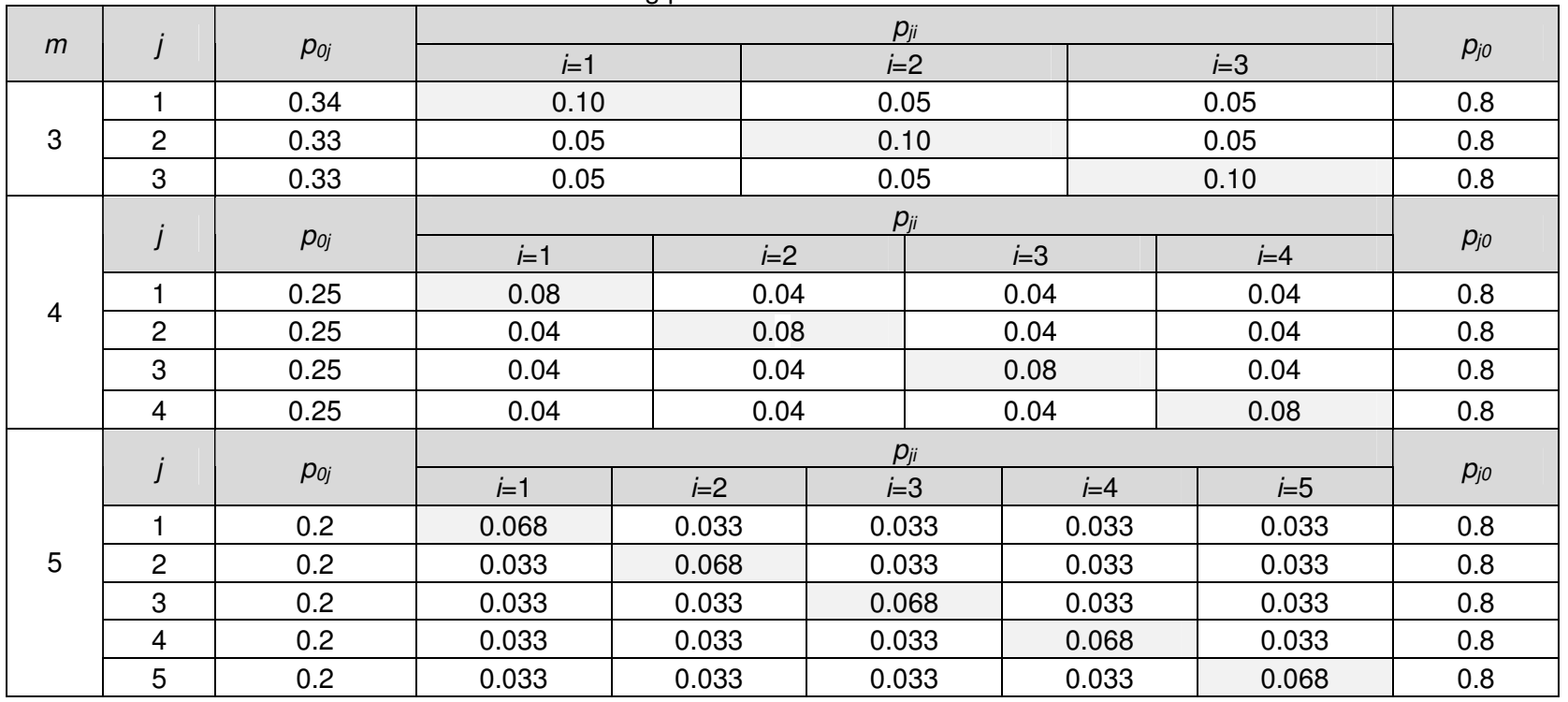

The results are shown in Figure 2. The main outcomes of these investigations are that $m$ has no effect on $U_{s}$, but $U_{j}$ decreases as $m$ increases, and both $U_{j}$ and $U_{s}$ are decreasing as $p_{d}(l)$ increases. This is because $U_{j}$ and $U_{s}$ represent a ratio between the number of packets left the router to the total number arrived, and as $p_{d}(J)$ increases the total number of packets dropped increases, so that the total number of packets leaving the router decreases, and consequently $U_{i}$ and $U_{s}$ are decreased.

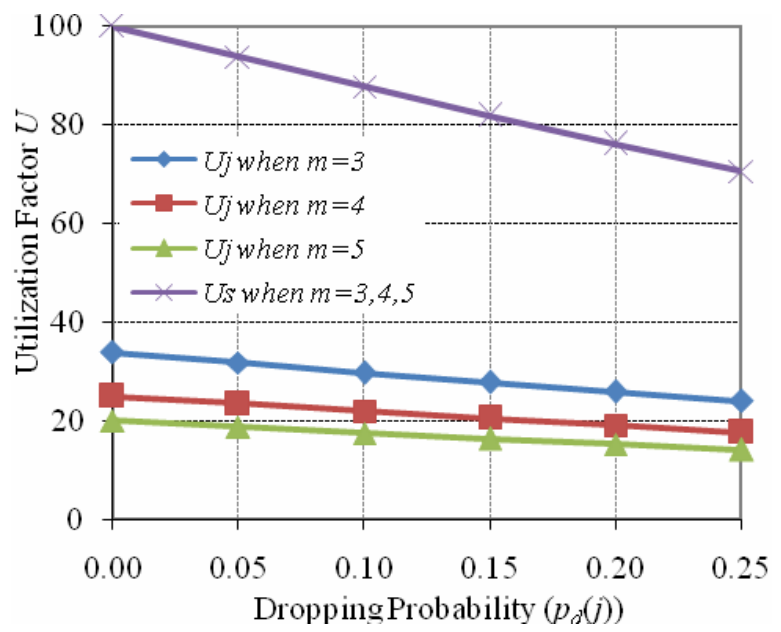

FIGURE 2: Variation of $U_{j}$ and $U_{s}$ against $p_{d}(J)$ for Scenario \#1.

\subsection{Scenario \#2. Investigate the Variation of $U_{j}$ and $U_{s}$ against $p_{d}(J)$ for Various Values of $p_{0 j}$}

In scenario \#2, the $m Q N$ model is used to investigate the variation of $U_{j}$ and $U_{s}$ against $p_{d}(J)$ for values of $p_{0 j}$. The values of $p_{d}(J)$ vary between 0 to 0.25 in step of 0.05 . The queuing system is assumed to have three queue nodes $(m=3)$ and $\lambda_{\text {arr }}=1$. Four cases are considered each with different arrival routing probabilities as given in Table 2. In addition, Table 2 tabulates other input parameters, namely, feedback, bypass, and departure routing probabilities. The results for this scenario are shown in Figure 3. 
TABLE 2: Packets routing probabilities for Scenario \#1

\begin{tabular}{|c|c|c|c|c|}
\hline .Case & $p_{01}$ & \multicolumn{2}{|c|}{$p_{02}$} & $p_{03}$ \\
\hline 1 & 0.6 & \multicolumn{2}{|c|}{0.3} & 0.1 \\
\hline 2 & 0.5 & \multicolumn{2}{|c|}{0.3} & 0.2 \\
\hline 3 & 0.5 & \multicolumn{2}{|c|}{0.25} & 0.25 \\
\hline 4 & 0.34 & \multicolumn{2}{|c|}{0.33} & 0.33 \\
\hline \multirow{2}{*}{$j$} & \multicolumn{3}{|c|}{$p_{j i}$} & \multirow{2}{*}{$p_{j 0}$} \\
\hline & $i=1$ & $i=2$ & $i=3$ & \\
\hline 1 & 0.10 & 0.05 & 0.05 & 0.8 \\
\hline 2 & 0.05 & 0.10 & 0.05 & 0.8 \\
\hline 3 & 0.05 & 0.05 & 0.10 & 0.8 \\
\hline
\end{tabular}
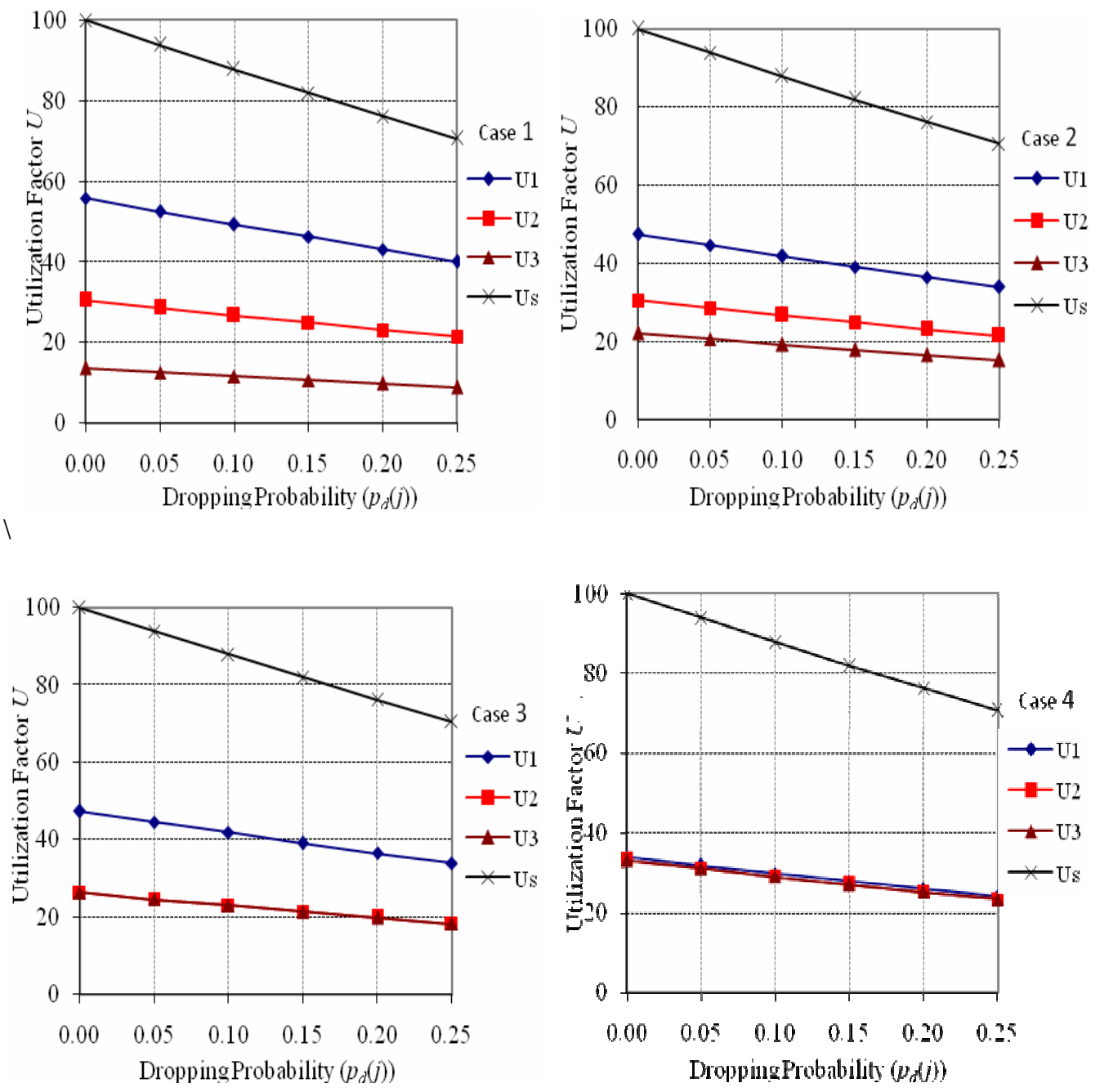

FIGURE 3: Variation of $U_{j}$ and $U_{s}$ against $p_{d}(J)$ for Scenario \#2.

The main outcomes of this set can be summarized as follows:

(1) For each set of $p_{0 j}, U_{j}$ and $U_{s}$ are decreasing linearly as $p_{d}(V)$ increases for the same reasons discussed in Scenario \#1.

(2) For a certain value of $p_{d}(\lambda), U_{s}$ is the same regardless of the values of $p_{0 j}$. For example, when $p_{d}(\lambda)=0.05, U_{s}=93.8 \%$ for all cases 1 to 4 , where each case presents different values of $p_{0 j}$. 
(3) For a certain value of $p_{d}(\lambda)$, the $U_{j}$ directly proportional to $p_{0 j}$.

(4) The system $U_{s}$ is the sum of the queue nodes $U_{j}$ as it was proved in Eqn. (15).

(5) The system presents an $U_{s}$ of $100 \%$, when $p_{d}(J)=0$.

\section{CONCLUSIONS}

Based on establishing a packet balance at the network router end-points, namely, the arrival and departure points, an analytical model was derived to evaluate the performance of a multi-queue nodes system designed to process packets arrived at the network router. The model was referred to as the mQN model. The derived expressions can be used effectively to accurately evaluate and investigate the performance of a multi-queue nodes system running under different network conditions and characterized by different design parameters. The cost of implementing and running the analytical model is minimal as compared to perform the same evaluation and investigation using computer simulations or experimental tests.

The analytical model proposed in this paper is expected to appreciably and significantly contribute to a number of future studies, such as: using the mQN models to perform more scenarios investigating the variation of the queue node and system performance for other combinations of input parameters. For example, investigate the effect of departure probabilities. It is highly recommended to dynamically change the routing probabilities as the number of connections and system load changes. In this case network routers can effectively control their resources to maintain high link utilization. This mechanism is attractive for the deployment of large IP networks supporting a wide range of connections and large aggregations of traffic.

\section{REFERENCES}

[1] L. Guan, I. U. Awan, M. E. Woodward, and X. Wang. Discrete-Time Performance Analysis of a Congestion Control Mechanism Based on RED under Multi-Class Bursty and Correlated Traffic. Journal of Systems and Software, Vol. 80, Issue 10, pp. 1716-1725, 2007.

[2] M. Welzl. Network Congestion Control: Managing Internet Traffic. John Wiley \& Sons, 2005.

[3] S. Floyd and V. Jacobson. Random Early Detection Gateways for Congestion Avoidance. IEEE/ACM Transactions on Networking, Vol. 1, No. 4, pp. 397-413, 1993.

[4] M. E. Woodward. Communication and Computer Networks: Modeling with Discrete-Time Queues. Pentech Press, 2005.

[5] H. Abdel-Jaber, F. Thabtah, M. E. Woodward, and Amir Abu-Ali. Performance Evaluation for DRED Discrete-Time Queuing Network Analytical Model. Journal of Network and Computer Applications, Vol. 31, Issue 4, pp. 750-770, November 2008.

[6] H. Abdel-Jaber, M. E. Woodward, F. Thabtah, and M. Etbega. A Discrete-Time Queue Analytical Model Based on Dynamic Random Early Drop. Proceedings of the $4^{\text {th }}$ IEEE International Conference on Information Technology: New Generations (ITNG 2007), Las-Vegas, USA, pp. 71-76, April 2007.

[7] J. Aweya, M.Ouellette, and D. Y. Montuno. A Control Theoretic Approach to Active Queue Management. Journal of Computer Networks, Vol. 36, Issue 2-3, pp. 203-235, 2001.

[8] T. G. Robertazzi. Computer Networks and Systems: Queuing Theory and Performance Evaluation. $3^{\text {rd }}$ Edition, Springer Verlag. 2000.

[9] H. Al-Bahadili. On the Use of Discrete-Event Simulation in Computer Networks Analysis and Design. Handbook of Research on Discrete-Event Simulation Environments: Technologies and Applications (Editors: Evon M. O. Abu-Taieh and Asim A. El-Sheikh), Information Science Reference, ISBN: 978-160566774-4, Chapter 19, 2009.

[10] J. Ababneh. Development of a Discrete-Time DRED-Based Multi-Queue Nodes Network Analytical Model. PhD Thesis, The Arab Academy for Banking \& Financial Sciences, Amman-Jordan, May 2009.

[11] L. Hoflack, S. De Vuyst, S. Wittevrongel, and H. Bruneel. System Content and Packet Delay in DiscreteTime Queues with Session-Based Arrivals. Proceeding of the $5^{\text {th }}$ International Conference on Information Technology: New Generations, pp. 1053-1058, 2008.

[12] F. Baccelli and D. R. McDonald. A Stochastic Model for the Throughput of Non-Persistent TCP Flows. Journal of Performance Evaluation, Vol. 65, Issues 6-7, pp. 512-530, 2008. 
[13] I. Awan, A. Yar, M. E. Woodward. Analysis of Queuing Networks with Blocking under Active Queue Management Scheme. Proceedings of the $12^{\text {th }}$ International Conference on Parallel and Distributed Systems (ICPADS'06), Vol. 2, pp. 61-68, 2006.

[14] L. Wang, G. Min, I. Awan. Modeling Active Queue Management with Different Traffic Classes. Proceedings of the $20^{\text {th }}$ International Conference on Advanced Information Networking and Applications, Vol. 2, pp. 442-446, 2006.

[15] M. Joshi, A. Mansata, S. Talauliker, and C. Beard. Design and Analysis of Multi-Level Active Queue Management Mechanisms for Emergency. Journal of Computer Communications, Vol. 28, Issue 2, pp. 162-173, 2005.

[16] W. H. Press, S. A. Teukolsky, W. T. Vetterling, and B. P. Flannery. The Art of Scientific Computing. $3^{\text {rd }}$ Edition, Cambridge University, 2007. 